\title{
Impedance Spectroscopy of OmpF Porin Reconstituted into a Hg-Supported Lipid Bilayer
}

Lucia Becucci, Maria Rosa Moncelli, and Rolando Guidelli*

Department of Chemistry, Florence University, Via della Lastruccia 3, 50019 Sesto Fiorentino

(Firenze), Italy

\section{Supporting Information}

Let $j_{+, \mathrm{i}}$ and $j_{-. \mathrm{i}}$ denote the current densities due to the flow of $\mathrm{K}^{+}$and $\mathrm{Cl}^{-}$ions towards the electrode within the generic $i$-th slab, with $i=1, . ., n$, and let $\Gamma_{+. \mathrm{i}}$ and $\Gamma_{-. \mathrm{i}}$ denote the $\mathrm{K}^{+}$and $\mathrm{Cl}^{-}$ surface concentrations at the boundary of the given slab on the metal side. The perturbations of these quantities are related by the equations:

$$
F \frac{\partial \Delta \Gamma_{+, i}}{\partial t}=-\Delta j_{+, i} \quad ; \quad F \frac{\partial \Delta \Gamma_{-, i}}{\partial t}=\Delta j_{-, i} \quad ; \quad \Delta j_{i}=\Delta j_{+, i}+\Delta j_{-, i}
$$

where $\Delta j_{\mathrm{i}}$ is the perturbation of the whole current density flowing across the $i$-th slab. The signs in the above equations account for the current due to a flow of positive (negative) charges toward the electrode being conventionally regarded as negative (positive). In view of the small a.c. perturbation, $\Delta E$, of the applied potential $E$, the dependence of $\Delta j_{\mathrm{h}, \mathrm{i}}$ and $\Delta \Gamma_{\mathrm{h}, \mathrm{i}}$ upon $\Delta E$ and the different $\Delta \Gamma_{\mathrm{h}, \mathrm{k}}$ 's, with $h=+,-$ and $k=1, \ldots, n$, can be linearized:

$$
\Delta j_{ \pm, i}=\frac{\partial j_{ \pm, i}}{\partial E} \Delta E+\sum_{h=+,-} \sum_{k=1}^{n} \frac{\partial j_{ \pm, i}}{\partial \Gamma_{h, k}} \Delta \Gamma_{h, k} \quad ; \quad \Delta \Gamma_{ \pm, l}=\frac{\partial \Gamma_{ \pm, \imath}}{\partial E} \Delta E+\sum_{\substack{h=+.-k=1 \\ k \neq i}}^{n} \frac{\partial \Gamma_{ \pm, \imath}}{\partial \Gamma_{h, k}} \Delta \Gamma_{h, k}(\mathrm{~A} 2)
$$

with $i=1, . ., n$. Here, the subscript \pm indicates that the given quantity refers, alternatively, to the cation and to the anion, and that the equation in which it appears is to be applied twice, first with the plus and then with the minus sign. Passing to the Laplace transforms of eqs.A1 and A2 and dividing both members of the resulting equations by the Laplace transform of $\Delta E, \mathcal{L}(\Delta E)$, we have:

$$
\begin{aligned}
& \frac{\mathcal{L}\left(\Delta j_{i}\right)}{\mathcal{L}(\Delta E)}=-s F\left[\frac{\mathcal{L}\left(\Delta \Gamma_{+, i}\right)}{\mathcal{L}(\Delta E)}-\frac{\mathcal{L}\left(\Delta \Gamma_{-, i}\right)}{\mathcal{L}(\Delta E)}\right] \\
& \frac{\mathcal{L}\left(\Delta j_{i}\right)}{\mathcal{L}(\Delta E)}=\frac{\partial j_{+, i}}{\partial E}+\frac{\partial j_{-, i}}{\partial E}+\sum_{h=+,-} \sum_{k=1}^{n}\left(\frac{\partial j_{+, i}}{\partial \Gamma_{h, k}}+\frac{\partial j_{-, i}}{\partial \Gamma_{h, k}}\right) \frac{\mathcal{L}\left(\Delta \Gamma_{h, k}\right)}{\mathcal{L}(\Delta E)} \\
& \frac{\partial \Gamma_{ \pm, i}}{\partial E}=\frac{\mathcal{L}\left(\Delta \Gamma_{ \pm, i}\right)}{\mathcal{L}(\Delta E)}-\sum_{h=+,-} \sum_{\substack{k=1 \\
k \neq i}}^{n} \frac{\partial \Gamma_{ \pm, i}}{\partial \Gamma_{h, k}} \frac{\mathcal{L}\left(\Delta \Gamma_{h, k}\right)}{\mathcal{L}(\Delta E)}
\end{aligned}
$$


with $i=1, \ldots, n$. If we replace the variable $s$ in eq. A3 by $i \omega$, where $i$ is the imaginary unit, in accordance with the usual procedure, then the $\mathcal{L}\left(\Delta j_{i}\right) / \mathcal{L}(\Delta E)$ ratio in this equation yields the quadrature component of the admittance. The corresponding ratio in eq.A4 yields the in-phase component; the $2 n \mathcal{L}\left(\Delta \Gamma_{h, k}\right) / \mathcal{L}(\Delta E)$ ratios on the right-hand side of eqs.A3 and A4 are obtained by solving the system of $2 n$ linear algebraic equations of eq.A5. The admittance of an $R C$ mesh has the form $\bar{g}_{\mathrm{i}}+i \omega \bar{C}_{\mathrm{i}}$. Consequently, we obtain:

$$
\begin{aligned}
& \bar{g}_{i}=\frac{\partial j_{+, i}}{\partial E}+\frac{\partial j_{-, i}}{\partial E}+\sum_{h=+,-} \sum_{k=1}^{n}\left(\frac{\partial j_{+, i}}{\partial \Gamma_{h, k}}+\frac{\partial j_{-, i}}{\partial \Gamma_{h, k}}\right) \frac{\mathcal{L}\left(\Delta \Gamma_{h, k}\right)}{\mathcal{L}(\Delta E)} \\
& \bar{C}_{i}=-F\left[\frac{\mathcal{L}\left(\Delta \Gamma_{+, i}\right)}{\mathcal{L}(\Delta E)}-\frac{\mathcal{L}\left(\Delta \Gamma_{-, i}\right)}{\mathcal{L}(\Delta E)}\right]
\end{aligned}
$$

These expressions of $\bar{g}_{i}$ and $\bar{C}_{\mathrm{i}}$ allow a semi-quantitative interpretation of the plots of these parameters against $E$ resulting from the fitting program. To this end, $j_{\mathrm{i}}$ must be expressed as a function of $\phi_{\mathrm{i}}$ and of the different surface concentrations $\Gamma_{\mathrm{h}, \mathrm{k}}$, with $h=+,-$ and $k=1, \ldots, n$.

For the system under study, the passage of $\mathrm{K}^{+}$ions from the bulk solution to the boundary between the lipid bilayer and the TEO moiety requires the surmounting of a potential energy barrier; the resulting current $j_{+.2}$ can be expressed by a Butler-Volmer-like equation:

$$
j_{+, 2}=-F\left\{\dot{k}_{+, 2}^{\prime} c_{K^{+}}\left(\Gamma_{m}-\Gamma_{+, 2}-\Gamma_{-, 2}\right) \exp \left(-\alpha f \phi_{2}\right)-\breve{k}_{+, 2} \Gamma_{+, 2} \exp \left[(1-\alpha) f \phi_{2}\right]\right\}
$$

with $f=\mathrm{F} / \mathrm{R} T$. Here, $c_{K^{+}}$is the bulk $\mathrm{K}^{+}$concentration, $\dot{k}_{+, 2}$ and $\breve{k}_{+, 2}$ are forward and backward rate constants for $\phi_{2}=0, \Gamma_{\mathrm{m}}$ is the maximum surface concentration attainable at the boundary between the lipid bilayer and the TEO moiety, and $\alpha$ is the charge transfer coefficient, which usually assumes values close to 0.5 . Under equilibrium conditions, $j_{+, 2}$ equals zero, yielding a Langmuirtype adsorption isotherm for $\mathrm{K}^{+}$ions at the boundary between the lipid bilayer and the TEO moiety:

$$
\frac{\Gamma_{+, 2}}{c_{K^{+}}\left(\Gamma_{m}-\Gamma_{+, 2}-\Gamma_{-, 2}\right)}=K_{+, 2} \exp \left(-f \phi_{2}\right) \quad \text { with }: \quad K_{+, 2} \equiv \frac{\dot{k_{+}}}{k_{+, 2}}
$$

Similarly, the current due to the translocation of $\mathrm{Cl}^{-}$ions across the lipid bilayer is expressed by the equation:

$$
j_{-, 2}=F\left\{k_{-, 2}^{\prime} c_{C l^{-}}\left(\Gamma_{m}-\Gamma_{+, 2}-\Gamma_{-, 2}\right) \exp \left(\alpha f \phi_{2}\right)-\breve{k}_{-, 2} \Gamma_{-, 2} \exp \left[-(1-\alpha) f \phi_{2}\right]\right\}
$$

which, at equilibrium, becomes: 
$\frac{\Gamma_{-, 2}}{c_{C l^{-}}\left(\Gamma_{m}-\Gamma_{+, 2}-\Gamma_{-, 2}\right)}=K_{-, 2} \exp \left(f \phi_{2}\right) \quad$ with $: \quad K_{-, 2} \equiv \frac{\dot{k}_{s} \cdot 2}{k_{-, 2}}$

It should be noted that the equilibrium constant $\mathrm{K}_{+, 2}$ includes any difference between the "formal" chemical potential, $\mu_{+, 2}^{0}$, of $\mathrm{K}^{+}$ions in the hydrophilic spacer and that, $\mu_{+, b}^{0}$, in the bulk solution. In fact, at equilibrium, the electrochemical potential of $\mathrm{K}^{+}$ions is the same in the aqueous solution and in the TEO moiety:

$\mu_{+, b}^{0}+R T \ln c_{K^{+}}=\mu_{+, 2}^{0}+R T \ln \frac{\Gamma_{+, 2}}{\Gamma_{m}-\Gamma_{+, 2}-\Gamma_{-, 2}}+F \phi_{2}$

It follows that:

$$
K_{+, 2}=\exp \left[-\left(\mu_{+, 2}^{0}-\mu_{+, b}^{0}\right) / R T\right]
$$

The two formal chemical potentials include both ion-ion interactions not embodied in the average potential difference $\phi_{2}$ and interactions of the $\mathrm{K}^{+}$ions with the main component of the respective phase. An analogous expression holds for the equilibrium constant $\mathrm{K}_{-, 2}$ :

$$
K_{-, 2}=\exp \left[-\left(\mu_{-, 2}^{0}-\mu_{-, b}^{0}\right) / R T\right]
$$

By analogous arguments, the current $j_{+.1}$ due to the translocation of $\mathrm{K}^{+}$ions across the TEO moiety is expressed by the equation:

$$
j_{+, 1}=-F\left\{k_{+, 1}^{\prime} \Gamma_{+, 2}\left(\Gamma_{m}-\Gamma_{+, 1}-\Gamma_{-, 1}\right) \exp \left(-\alpha f \phi_{1}\right)-\breve{k}_{+, 1} \Gamma_{+, 1}\left(\Gamma_{m}-\Gamma_{+, 2}-\Gamma_{-, 2}\right) \exp \left[(1-\alpha) f \phi_{1}\right]\right\}
$$

Here, $\dot{k}_{+, 1}$ and $\breve{k}_{+, 1}$ are forward and backward rate constants for $\phi_{1}=0$, while $\Gamma_{\mathrm{m}}$ is the maximum surface concentration attainable at the boundary between the TEO moiety and the lipoic acid residue, regarded as equal to that at the boundary between the lipid bilayer and the TEO moiety. This assumption is based on the consideration that, at saturation, $\mathrm{K}^{+}$ions are distributed throughout the whole hydrophilic portion of the thiolipid. Since the ideal circuit elements used for the fitting represent ideal lumped-constant properties, the assumption of the same maximum surface concentration at both boundaries of the TEO moiety is justified. At equilibrium, eq.A15 reduces to a Langmuir-type adsorption isotherm for $\mathrm{K}^{+}$ions at the boundary between the TEO moiety and the lipoic acid residue:

$$
\frac{\Gamma_{+, 1}\left(\Gamma_{m}-\Gamma_{+, 2}-\Gamma_{-, 2}\right)}{\Gamma_{+, 2}\left(\Gamma_{m}-\Gamma_{+, 1}-\Gamma_{-, 1}\right)}=K_{+, 1} \exp \left(-f \phi_{1}\right) \quad \text { with }: K_{+, 1} \equiv \frac{\dot{k}_{\mathbf{S}^{+, 1}}}{k_{+, 1}}
$$

Likewise, the current due to the translocation of $\mathrm{Cl}^{-}$ions across the TEO moiety is given by the equation: 


$$
j_{-, 1}=F\left\{\dot{k}_{-, 1}^{\prime} \Gamma_{-, 2}\left(\Gamma_{m}-\Gamma_{+, 1}-\Gamma_{-, 1}\right) \exp \left(\alpha f \phi_{1}\right)-\breve{k}_{-, 1} \Gamma_{-, 1}\left(\Gamma_{m}-\Gamma_{+, 2}-\Gamma_{-, 2}\right) \exp \left[-(1-\alpha) f \phi_{1}\right]\right\}
$$

which, at equilibrium, reduces to the Langmuir isotherm:

$$
\frac{\Gamma_{-, 1}\left(\Gamma_{m}-\Gamma_{+, 2}-\Gamma_{-, 2}\right)}{\Gamma_{-, 2}\left(\Gamma_{m}-\Gamma_{+, 1}-\Gamma_{-, 1}\right)}=K_{-, 1} \exp \left(f \phi_{1}\right) \quad \text { with }: K_{-, 1} \equiv \frac{\dot{k}_{S}^{-1}}{k_{-, 1}}
$$

The dependence of $\phi_{1}$ and $\phi_{2}$ upon $E, \Gamma_{ \pm, 1}$ and $\Gamma_{ \pm, 2}$, is obtained from eq. 1. Expressing $q$ as a function of $E$ and of the four surface concentrations through eq. 1 and replacing it into the expressions for $\phi_{1}$ and $\phi_{2}$ yields:

$$
\begin{aligned}
& \phi_{1}=\frac{\left(E+0.25 V-\chi_{1}\right) C_{0} C_{2}+F\left(\Gamma_{+, 1}-\Gamma_{-, 1}\right) C_{2}-F\left(\Gamma_{+, 2}-\Gamma_{-, 2}\right) C_{0}}{C_{1} C_{2}+C_{0} C_{1}+C_{0} C_{2}}+\chi_{1} \\
& \phi_{2}=\frac{\left(E+0.25 V-\chi_{1}\right) C_{0} C_{1}+F\left(\Gamma_{+, 1}-\Gamma_{-, 1}\right) C_{1}+F\left(\Gamma_{+, 2}-\Gamma_{-, 2}\right)\left(C_{0}+C_{1}\right)}{C_{1} C_{2}+C_{0} C_{1}+C_{0} C_{2}}
\end{aligned}
$$

Values of $\Gamma_{ \pm, 2}$ and $\Gamma_{ \pm, 1}$ at constant applied potential are obtained from their adsorption isotherms (eqs.A9, A11, A16 and A18), in which $\phi_{1}$ and $\phi_{2}$ are expressed by eq.A19, using the Newton-Raphson iterative procedure. The derivatives of the four surface concentrations are then substituted into eq.A5 and the system of the four linear algebraic equations is solved to obtain the $\mathcal{L}\left(\Delta \Gamma_{h, k}\right) / \mathcal{L}(\Delta E)$ ratios; their substitution into eq.A7 yields the capacities $\bar{C}_{1}$ and $\bar{C}_{2}$ of the TEO moiety and of the lipid bilayer, respectively. The values of the surface concentrations are then substituted into the expressions for $j_{ \pm}, 2$ and $j_{ \pm, 1}$ of eqs.A8, A10, A15, and A17, and the derivatives of these currents are replaced into eq.A6 to obtain the conductivities $\bar{g}_{1}$ and $\bar{g}_{2}$ of the TEO moiety and of the lipid bilayer. It is evident that the $\bar{C}_{1}$ and $\bar{C}_{2}$ values so obtained as a function of $E$ depend only on the equilibrium constants of eqs. A9, A11, A16 and A18, and not on the kinetic parameters; hence, they express the system behavior at low frequencies.

This is more clearly apparent if we consider, as an example, a single isolated dielectric slab subjected to a current perturbation $\Delta j$ and we apply eqs.A1 and A2:

$$
\Delta j=-F \frac{\partial \Delta \Gamma}{\partial t}=\frac{\partial j}{\partial \Gamma} \Delta \Gamma+\frac{\partial j}{\partial E} \Delta E
$$

Passing to the Laplace transforms of these equations, replacing the parameter $s$ of the transformation by $i \omega$, and rearranging terms, we obtain the following expression for the admittance of the slab:

$$
\frac{\mathcal{L}(\Delta j)}{\mathcal{L}(\Delta E)}=\frac{\omega^{2} F^{2}(\partial j / \partial E)}{\omega^{2} F^{2}+(\partial j / \partial \Gamma)^{2}}+i \omega F \frac{(\partial j / \partial \Gamma)(\partial j / \partial E)}{\omega^{2} F^{2}+(\partial j / \partial \Gamma)^{2}}
$$


If we compare this expression with the admittance of a $R C$ mesh, $\bar{g}+i \omega \bar{C}$, we immediately realize that the in-phase component of eq.A21 yields a frequency-independent conductance $\bar{g}=(\partial j / \partial E)$ for the $R C$ mesh when $(\partial j / \partial \Gamma)$ is $<<\omega \mathrm{F}$, namely at high frequencies. Conversely, the quadrature component yields a frequency-independent capacity $\bar{C}=\mathrm{F}(\partial j / \partial E) /(\partial j / \partial \Gamma)=-\mathrm{F}(\partial \Gamma / \partial E)$ for the mesh when $(\partial j / \partial \Gamma)$ is $>>\omega \mathrm{F}$, namely at low frequencies. Note that the above expressions for $\bar{g}$ and $\bar{C}$ are analogous to the more general expressions of eqs. A6 and A7.

Since the conductivities $\bar{g}_{1}$ and $\bar{g}_{2}$ refer to high frequencies, in estimating them the differentiation of $\Gamma_{ \pm, 1}$ and $\Gamma_{ \pm, 2}$ in eq.A5 with respect to $E$ was carried out by ignoring the implicit dependence of $\phi_{1}$ and $\phi_{2}$ upon $\Gamma_{ \pm, 1}$ and $\Gamma_{ \pm, 2}$, as expressed by eq. A19. This amounts to assuming that a change in $E$ is fast enough to affect $\Gamma_{ \pm, 1}$ and $\Gamma_{ \pm, 2}$ without having a feedback effect on $\phi_{1}$ and $\phi_{2}$. By repeating this procedure at all potentials, plots of the latter quantities against $E$ can be obtained. 